\title{
Varistor-like current-voltage characteristic of porous silicon
}

\author{
O.V. Vakulenko, S.V. Kondratenko, B.M. Shutov . \\ Taras Shevchenko Kyiv Univ., 6 Glushkova Prosp., 252127 Kyiv, Ukraine \\ Tel.80442660162,E-mail: kondr@hq.ups.kiev.ua
}

\begin{abstract}
The porous silicon (PS) current-voltage characteristic (CVC) has measured in transverse and longitudinal applied electric field. The obtained CVC has a varistor-like shape. Besides the practical application this confirms the PS grain structure whose influence is discussed in analizing the mechanism of the visible luminescence in PS.
\end{abstract}

Keywords: varistor, current-voltage characteristic, porous silicon.

Paper received 25.06.99; revised manuscript received 06.07.99; accepted for publication 12.07.99.

\section{Introduction}

The notion of the nanocrystallites in randomly positioned quantum wires or quantum walls between neighbouring pores of the porous silicon (PS) samples has been applied by the reseachers to explain a lot of photophysical effects, the visible photoluminescence in particular [1-3]. The availability of such grain structure may also manifest itself in the PS electrical properties. Hopefully, an asymmetric varistor-like current-voltage characteristic (CVC) can be observed in the structures with porous silicon layer. However, only asymmetric diode-like CVC has been reported [4-7]. So, the question about the existence of such grain structure could be supplementary verified if one succeeded in finding varistor-like CVC in the porous silicon samples. This has been the subject of our work. The problem of measuring CVC of PS in longitudinal electric field, when both contacts are applied to the PS side, lies in the fact that only PS layer has a larger resistance in comparision with $\mathrm{Si}$ substrate. Consequently, one should expect a short out effect by the material of Si substrate. However, we succeeded in preparing the PS layers without the short out effect. These layers in the multilayer structure of the PS, as all the others, consisted of Si nanocrystallites in $\mathrm{SiO}_{\mathrm{x}}(0<\mathrm{x}<2)$ matrix, but differed from others by moderate oxidation. It is this moderate oxidation that favoured the conductivity enhancement of the relevant sublayer of the sample, as it has been reported [2,3]. Therefore, the partially oxidated layers in PS have been investigated. In addition, these layers showed a high-intensity visible photoluminescence.

\section{Experiment}

The porous silicon samples were prepared using anodization in $48 \%$ HF solution with a current density $25 \mathrm{~mA} / \mathrm{cm}^{2}$ for ten minutes. (111) - oriented $p$-type silicon wafers were used for substrates. Before anodization the substrates were cleaned, and the ohmic contact was formed in a thin Al layer on the back side of the Si substrate. The PS layer thickness was approximately $5 \mu \mathrm{m}$. After anodizing a few semitransparant Au point contacts have been deposited onto the PS surface (Fig.1). This made it possible to observe con-

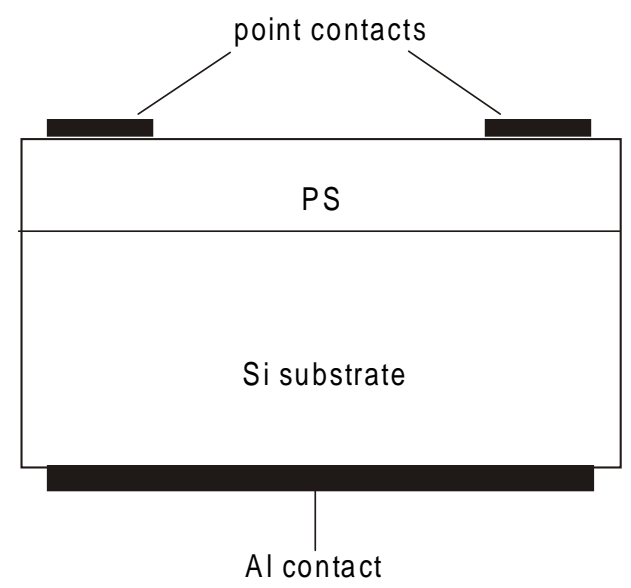

Fig. 1. Structure with PS layer for CVC measurements. 


\section{O.V. Vakulenko et al.: Varistor-like current-voltage characteristic of porous silicon}

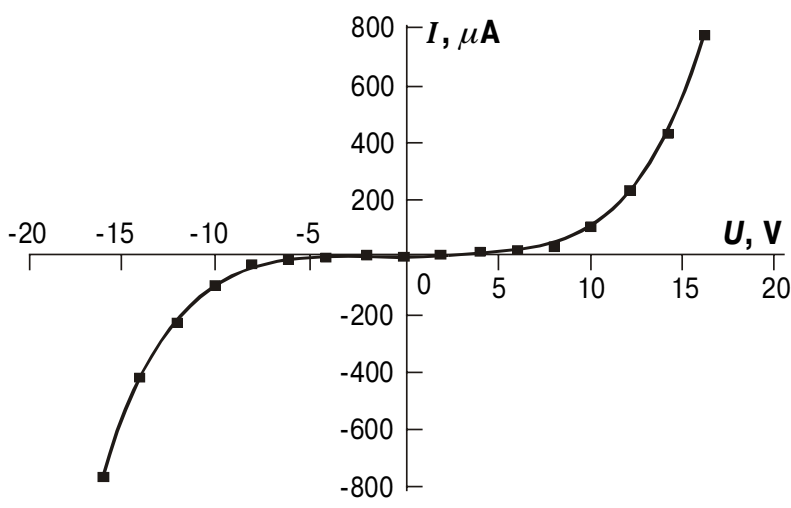

Fig. 2. Varistor-like CVC of the PS samples observed in longitudinally applied electric field.

ductivity in transverse and longitudinal applied electric field in relation to the PS layer.

\section{Results and discussion}

Asymmetric diode-like current-voltage characteristic is always observed in transverse electric field measurments, because the porous silicon layer and the Si substrate give rise to a rectifying contact.

According to the transverse field measurments, our samples have been divided into two groups. For the first one, the current-voltage characteristic is similar to that for two oppositely switched diodes. This testifies that metal - PS contact forms a potential wall. The other group of the samples exhibited a symmetric varistor-like CVC (Fig.2). The $\mathrm{CVC}$ of these samples can be described by the equation: $I=B \cdot U^{n}$, where B is the sample constant depending on a lot of factors: preparation and storage conditions and many others. Here $\mathrm{n}$ is the varistor nonlinearity coefficient. For the majority of PS samples it varies from 4 to 5 . The CVC of the sample showed in Fig. 2 exhibits the magnitude of $n \approx 4.2$.

The varistor-like CVC points to the fact that potential wall on the PS - Au contact interface is absent for this type of samples. The PS material nonlinearity is usually attributed to the varistor conductivity enhancement in increasing the electric field intensity. In our case, it may be explained by the following reasons: emission of electrons from grain edges of the nanocrystallite structure formed in PS; large-field conductivity enhacement of the oxide film which covers the pores; microheating of the contacting points between pores, etc.

The PS material irregularity does not make it possible to choose one of the suggested reasons. However, the fact of existence of varistor-like CVC, besides the practical application, is of interest, because it favours the grain, nanocrystallite PS structure, whose influence is usually discussed in analyzing the mechanisms of the visible photoluminescence in PS.

\section{References}

1. S.V. Svechnikov, A.V. Sachenko, G.A. Sukach, A.M. Yevstigneyev, E.B. Kaganovich. Optoelektronika i poluprovodnikovaya tekhnika, 27, p. 3-29 (1994) (in Russian).

2. V.G. Golubev, A.V. Zherzdnev, G.K. Moroz, A.V. Patsekin, D.T. Yan. Fizika i tekhnika poluprovodnikov, 30(5), p. 852-863 (1996) (in Russian).

3. Y.Kanemitsu. Phys. Rev.B. 48(7),p. 4883-4886 (1993)

4. T.Ozaki, M.Araki, S.Yoshimura, H.Koyama, N.Koshida. J. Appl. Phys. 76(3), p.1986-1988 (1994).

5. L.V. Beliakov, D.N. Goriachev, O.M. Sreseli, I.D. Yaroshetski. Fizika i tekhnika poluprovodnikov, 27(8), p.1371-1375 (1993) (in Russian).

6. F.Namavar, H.P.Maruska, N.M.Khoran. Appl. Phys. Lett. 60(20), p.2514-2516 (1992).

7. N.Koshida, M.Koyama, Appl. Phys. Lett. 60(3), p.347-349 (1992). 